\title{
Null paths on a toroidal topological black hole in conformal Weyl gravity
}

\author{
J. R. Villanueva ${ }^{1, \mathrm{a}}$, Francisco Tapia ${ }^{1, \mathrm{~b}}$, Martín Molina ${ }^{1, \mathrm{c}}$, Marco Olivares ${ }^{2, \mathrm{~d}}$ \\ ${ }^{1}$ Instituto de Física y Astronomía, Universidad de Valparaíso, Avenida Gran Bretaña 1111, Playa Ancha, Valparaíso, Chile \\ ${ }^{2}$ Facultad de Ingeniería y Ciencias, Universidad Diego Portales, Avenida Ejército Libertador 441, Casilla 298-V, Santiago, Chile
}

Received: 13 August 2018 / Accepted: 9 October 2018 / Published online: 23 October 2018

(C) The Author(s) 2018

\begin{abstract}
The motion of massless particles on the background of a toroidal topological black hole is analyzed in the context of conformal Weyl gravity. Null geodesics, in terms of the Jacobi elliptic functions, are found analytically. In addition, the Sagnac effect in this space-time is characterized, and we find a strong condition in the theory's parameters that is required for its existence.
\end{abstract}

\section{Contents}

1 Introduction: conformal Weyl gravity and null geodesics ...................... 1

2 Toroidal topology .............. 2

3 Radial motion . . . . . . . . . . . . 3

4 Angular motion . . . . . . . . . . . . 3

5 The Sagnac effect . . . . . . . . . . . 6

6 Summary ................... . . 7

Appendix A: A brief review of Jacobian elliptic func-

tions ............... 7

References................ . . 8

\section{Introduction: conformal Weyl gravity and null geodesics}

Conformal Weyl gravity (CWG) was born of an attempt to unify gravity and electromagnetism based on the principle of local invariance of a manifold, described by the metric $g_{\mu \nu}(x)$, under the change

$g_{\mu \nu}(x) \rightarrow \Omega^{2}(x) g_{\mu \nu}(x)$,

a e-mail: jose.villanueva@uv.cl

b e-mail: fjtapiam@gmail.com

c e-mail: martin.molina@alumnos.uv.cl

d e-mail: marco.olivaresr@mail.udp.cl where $\Omega(x)$ is a smooth, strictly positive function [1-4]. The CWG theory can be obtained from the conformally invariant action

$I_{W}=2 \alpha_{w} \int \mathrm{d}^{4} x \sqrt{-g}\left[R_{\mu \nu} R^{\mu \nu}-\frac{1}{3}\left(R_{\mu}^{\mu}\right)^{2}\right]$,

where $\alpha_{w}$ is a dimensionless parameter chosen to be positive if Eq. (2) is a positive definite Euclidean action. The vacuum field equations associated with this action are solved by the static, spherically symmetric line element given by [5-9]

$\mathrm{d} \tilde{s}^{2}=-B(\tilde{r}) \mathrm{d} \tilde{t}^{2}+\frac{\mathrm{d} \tilde{r}^{2}}{B(\tilde{r})}+\tilde{r}^{2}\left(\mathrm{~d} \tilde{\theta}^{2}+\sin ^{2} \tilde{\theta} \mathrm{d} \tilde{\phi}^{2}\right)$,

where the coordinates are defined in the range $-\infty<\tilde{t}<\infty$, $\tilde{r} \geq 0,0 \leq \tilde{\theta} \leq \pi, 0 \leq \tilde{\phi} \leq 2 \pi$, and the lapse function $B(\tilde{r})$ is given by

$B(\tilde{r})=1-\frac{\tilde{\beta}(2-3 \tilde{\beta} \tilde{\gamma})}{\tilde{r}}-3 \tilde{\beta} \tilde{\gamma}+\tilde{\gamma} \tilde{r}-\tilde{k} \tilde{r}^{2}$

Here $\tilde{\beta}, \tilde{k}$ and $\tilde{\gamma}$ are positive constants associated with the central mass, cosmological constant and the measurements of the departure of the Weyl theory from the Einstein-de Sitter, respectively. Clearly, taking the limit $\tilde{\gamma}=0=\tilde{k}$ recovers the Schwarzschild case, so that we can identify $\tilde{\beta}=M$.

A study of the basis and properties, together with applications of the motion of massive and massless particles in this geometry can be found, for example, in [10-19], and can be obtained using the standard Lagrange procedure [20-27], which allows a Lagrangian $\mathscr{L}$ to be associated with the metric and then the equation of motion to be obtained from the Lagrange's equations,

$\dot{\Pi}_{q}-\frac{\partial \mathscr{L}}{\partial q}=0$ 
where $\Pi_{q}=\partial \mathscr{L} / \partial \dot{q}$ are the conjugate momenta to the coordinate $q$, and the dot denotes a derivative with respect to the affine parameter $\tau$ along the geodesic. Thus, in Sect. 2, and following the procedure performed by Klemm [28], we perform analytical continuations to obtain a non-trivial topology associated with toroidal topological black holes coming from CWG. In particular, we focus on the toroidal AdS black hole. Other studies associated with topological black holes can be found, for example, in Refs. [29-36]. Then we obtain the conserved quantities together with the equations of motion for massless particles on these manifolds. In Sect. 3 the radial motion is analyzed for photons going to spatial infinity or to the singularity, while Sect. 4 is devoted to obtaining analytically the trajectory for photons with non-zero angular momentum, for which we employ an analysis in terms of Jacobi elliptic functions. In Sect. 5 we apply the methods outlined by Sakurai, Tartaglia, Rizzi and Ruggiero, among others, to obtain an analogy to the Aharanov-Bohm effect to describe the Sagnac effect for this space-time. Finally, in Sect. 6 we conclude and summarize our results.

\section{Toroidal topology}

In order to obtain a toroidal topological black hole, we perform the following analytical continuation of the metric Eq. (3):

$$
\begin{aligned}
& \tilde{t} \rightarrow \sqrt{\alpha} t, \quad \tilde{r} \rightarrow \frac{r}{\sqrt{\alpha}}, \quad \tilde{\phi} \rightarrow \phi, \\
& \tilde{\theta} \rightarrow \sqrt{\alpha} \theta, \quad \tilde{\beta} \rightarrow \frac{\beta}{\sqrt{\alpha}}, \quad \tilde{\gamma} \rightarrow \frac{\gamma}{\sqrt{\alpha}}, \quad \tilde{k} \rightarrow k .
\end{aligned}
$$

Then, by taking the limit $\alpha \rightarrow 0$, the line element becomes

$$
\mathrm{d} s^{2}=-B(r) \mathrm{d} t^{2}+\frac{\mathrm{d} r^{2}}{B(r)}+r^{2}\left(\mathrm{~d} \theta^{2}+\theta^{2} \mathrm{~d} \phi^{2}\right),
$$

with the lapse function

$$
B(r)=\frac{3 \beta^{2} \gamma}{r}-3 \beta \gamma+\gamma r-k r^{2}
$$

In this case, it is possible to prove that the metric induced on the space-like surface of constant $t$ and $r$ corresponds to a compact orientable surface with genus $g=1$, i.e., a torus, so the topology of this four-dimensional manifold becomes $\mathbb{R}^{2} \times S^{1} \times S^{1}$ [28]. Therefore, performing $\gamma=-2 \eta / L$, $\beta=\sqrt{L / 3}, k=-1 / \ell^{2}$, and then evaluating at the limit $L \rightarrow \infty$, the lapse function becomes

$$
B(r)=-\frac{2 \eta}{r}+\frac{r^{2}}{\ell^{2}}
$$

which for $\eta>0$ describes the AdS uncharged static toroidal black hole [37-43] with the event horizon placed at

$r_{+}=\left(2 \eta \ell^{2}\right)^{1 / 3}$

The vanished Lagrangian associated with the photons that move on this manifold can be expressed as

$\mathscr{L}=-\frac{1}{2} B(r) \dot{t}^{2}+\frac{1}{2} \frac{\dot{r}^{2}}{B(r)}+\frac{1}{2} r^{2}\left(\dot{\theta}^{2}+\theta^{2} \dot{\phi}^{2}\right)=0$.

Conversely, the toroidal metric given by Eqs. (6) and (8) admits the following Killing vector field:

- The time-like Killing vector $\chi=\partial_{t}$ is related to the stationarity of the metric. The conserved quantity is given by

$g_{\mu \nu} \chi^{\mu} u^{\nu}=-B(r) \dot{t}=-\sqrt{E}$

where $E$ is a constant of motion that cannot be associated with the total energy of the test particle because this metric is not asymptotically flat.

- The most general space-like Killing vector is given by

$$
\begin{aligned}
\chi= & (A \cos \phi+B \sin \phi) \partial_{\theta} \\
& +\left[C-A \frac{\sin \phi}{\theta}+B \frac{\cos \phi}{\theta}\right] \partial_{\phi},
\end{aligned}
$$

where $A, B$ and $C$ are arbitrary constants. Is easy to see that it is a linear combination of the three Killing vectors

$$
\begin{aligned}
& \chi_{1}=\partial_{\phi}, \\
& \chi_{2}=\cos \phi \partial_{\theta}-\frac{\sin \phi}{\theta} \partial_{\phi}, \\
& \chi_{3}=\sin \phi \partial_{\theta}+\frac{\cos \phi}{\theta} \partial_{\phi}
\end{aligned}
$$

which are the angular momentum operators for this space-time. The conserved quantities are given by

$g_{\alpha \beta} \chi_{1}^{\alpha} u^{\beta}=r^{2} \theta^{2} \dot{\phi}=L_{1}$,

$g_{\alpha \beta} \chi_{2}^{\alpha} u^{\beta}=r^{2}(\cos \phi \dot{\theta}-\theta \sin \phi \dot{\phi})=L_{2}$,

$g_{\alpha \beta} \chi_{3}^{\alpha} u^{\beta}=r^{2}(\sin \phi \dot{\theta}+\theta \cos \phi \dot{\phi})=L_{3}$,

where $L_{1}, L_{2}$ and $L_{3}$ are constants associated with the angular momentum of the particles.

It is interesting to point out that Eqs. (14) and (15) imply that

$J^{2} \equiv L_{2}^{2}+L_{3}^{2}=r^{4}\left(\dot{\theta}^{2}+\theta^{2} \dot{\phi}^{2}\right)$, 


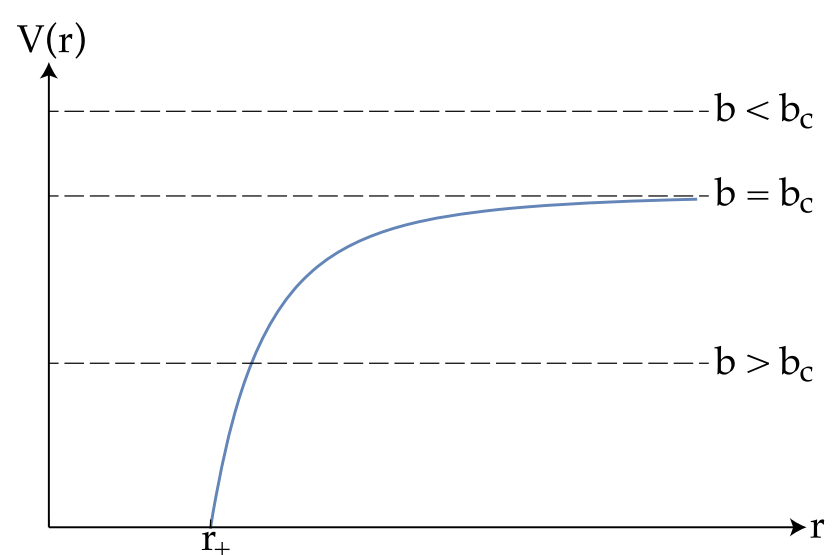

Fig. 1 Graphs of the topological toroidal effective potential as a function of the radial coordinate. The critical impact parameter $b_{c}$ corresponds to the value of $b$ at $r \rightarrow \infty$

such that, if we focus our attention on the invariant plane $\theta=\theta_{0}$, so $\dot{\theta}=0$, we get $L_{1}=\theta_{0} J$. Thus, using Eqs. (11) and (16) in Eq. (10) we obtain the radial equation of motion corresponding to the one-dimensional problem

$\dot{r}^{2}=E-\mathscr{V}(r)$,

where $\mathscr{V}(r)$ is the effective potential defined as

$\mathscr{V}(r)=J^{2} \frac{B(r)}{r^{2}} \equiv J^{2} V(r)$.

Here $V(r)=B(r) / r^{2}$ is the effective potential per unit of $J^{2}$.

On the other hand, without lack of generality we choose $\theta_{0}=1$ and combining Eqs. (16) and (17), we obtain the angular motion equation

$$
\left(\frac{1}{r^{2}} \frac{d r}{d \phi}\right)^{2}=\frac{1}{b^{2}}-V(r)=\frac{2 \eta}{r^{3}}-\left(\frac{1}{\ell^{2}}-\frac{1}{b^{2}}\right),
$$

where $b=J / \sqrt{E}$ is the impact parameter.

In Fig. 1 we have plotted the effective potential (per unit of $J^{2}$ ) as a function of the radial coordinate. In the next sections the motion of massless particles will be analyzed analytically by integration of the equations of motion.

\section{Radial motion}

Radial motion corresponds to a trajectory with null angular momentum $J=0$, so photons fall toward the singularity or to the spatial infinity. From Eq. (18) we can see that photons in radial motion have a null effective potential $\mathscr{V}(r)=0$, so that Eqs. (11) and (17) become $\frac{d r}{d \tau}= \pm \sqrt{E}$,

and

$\frac{\mathrm{d} r}{\mathrm{~d} t}= \pm B(r)= \pm \frac{1}{\ell^{2}}\left(\frac{r^{3}-r_{+}^{3}}{r}\right)$,

where the sign - $(+)$ corresponds to photons falling to the event horizon (spatial infinity). Assuming that $t=\tau=0$ at $r=r_{i}$, then a straightforward integration of Eq. (20) leads to

$\tau(r)= \pm \frac{r-r_{i}}{\sqrt{E}}$

while an integration of Eq. (21) becomes

$$
\begin{aligned}
t(r)= \pm & \frac{\ell^{2}}{\sqrt{3} r_{+}}\left\{\arctan \left(\frac{2 r+r_{+}}{\sqrt{3} r_{+}}\right)-\arctan \left(\frac{2 r_{i}+r_{+}}{\sqrt{3} r_{+}}\right)\right. \\
& \left.+\log \left[\frac{r_{i}^{3}-r_{+}^{3}}{r^{3}-r_{+}^{3}}\left(\frac{r-r_{+}}{r_{i}-r_{+}}\right)^{3}\right]\right\} .
\end{aligned}
$$

Obviously, in the proper system photons cross the event horizon in a finite time $\tau\left(r_{+}\right) \equiv \tau_{+}=\left(r_{i}-r_{+}\right) / \sqrt{E}$ and, eventually, arrive at the singularity in a finite time $\tau(0) \equiv \tau_{0}=r_{i} / \sqrt{E}$. Also, they eternally approach the spatial infinity i.e., $\tau(\infty) \rightarrow \infty$. On the other hand, an observer at $r_{i}$ sees that photons take an infinite coordinate time even to arrive at $r_{+}$, while it takes a finite coordinate time even to arrive at the spatial infinity, given by

$t_{\infty}=\frac{\ell^{2}}{\sqrt{3} r_{+}}\left\{\frac{\pi}{2}-\arctan \left(\frac{2 r_{i}+r_{+}}{\sqrt{3} r_{+}}\right)+\log \left[\frac{r_{i}^{3}-r_{+}^{3}}{\left(r_{i}-r_{+}\right)^{3}}\right]\right\}$.

The existence of this time is due to the presence of the cosmological term on the toroidal topology and depends on the position of the observer $r_{i}$. A similar feature was reported before by Villanueva and Vásquez, but in the context of Lifshitz black holes [44]. The behavior of both proper time and coordinate time is shown in Fig. 2.

\section{Angular motion}

This section is devoted to studying the angular motion of the test particles $(J \neq 0)$, which depends on the value of the impact parameter $b$. From Fig. 1 we can see that there are two distinct regions separated by the critical impact parameter 


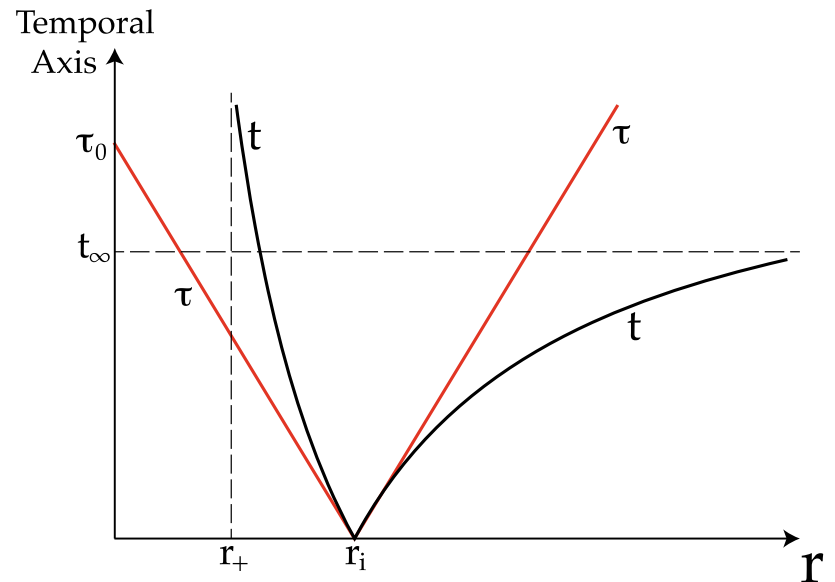

Fig. 2 Temporal null geodesics for radial motion described by Eqs. (22) and (23). This shows some equivalence with its spherical counterpart previously studied by Cruz et al. [21] for photons moving to $r_{+}$. The novel result appears for photons moving to infinity in which, as is measured by an observer in $r_{i}$, it takes a finite coordinate time $t_{\infty}$ to reach infinity. This feature was reported before by Villanueva and Vásquez but in the context of Lifshitz space-times [44]

$b_{c}=\ell$. Thus, if $b>b_{c}$ the motion will be confined with a turning point, the apoastron distance $r_{t}$, placed at

$r_{t}(b)=\frac{r_{+}}{\left[1-\left(\frac{b_{c}}{b}\right)^{2}\right]^{1 / 3}}$,

whereas if $b<b_{c}$ the turning point does not exist, and the motion is unbound with a negative characteristic distance (without physical meaning), the magnitude of which is

$r_{D}(b)=\frac{r_{+}}{\left[\left(\frac{b_{c}}{b}\right)^{2}-1\right]^{1 / 3}}$.

Both distances, $r_{t}$ and $r_{D}$, play an important role in the determination of trajectories because they depend strongly on the impact parameter $b$ (see Figs. 3 and 5).

\subsection{Confined motion}

Returning to the general equation (19), we first consider the case when the impact parameter lies between $b_{c}<b<\infty$, so using the variable $u=1 / r$ with $u_{t}=1 / r_{t}$, we can write

$$
\begin{aligned}
\frac{\mathrm{d} u}{\mathrm{~d} \phi} & = \pm \sqrt{2 \eta} \sqrt{u^{3}-u_{t}^{3}} \\
& = \pm \sqrt{2 \eta} \sqrt{\left(u-u_{t}\right)\left[\left(u+\frac{u_{t}}{2}\right)^{2}+\frac{3 u_{t}^{2}}{4}\right]} .
\end{aligned}
$$

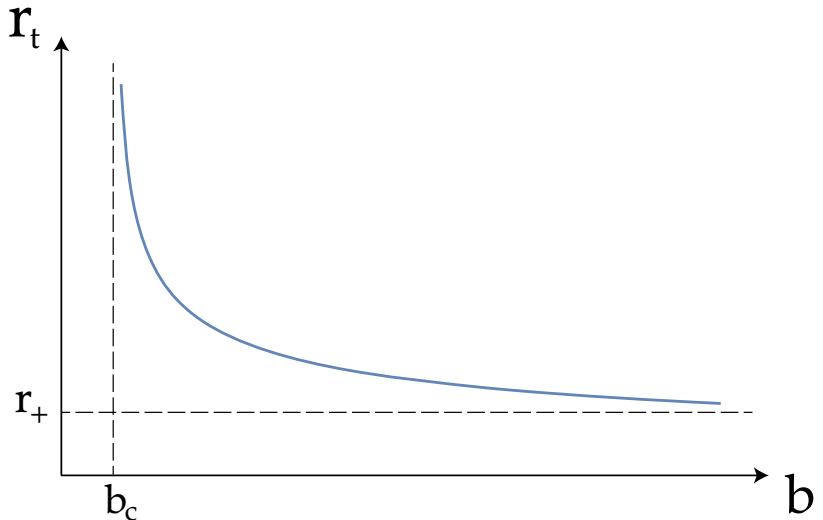

Fig. 3 Plot for the turning point or apoastron, $r_{t}$ as a function of the impact parameter $b$. The validity of this function is in $b_{c}<b<\infty$

Using the substitution

$u=u_{t}\left(\sqrt{3} \cot ^{2} \frac{\xi}{2}+1\right)$

where the range of $u$ is $u_{t} \leq u<\infty$, and the corresponding range of $\xi$ is $\xi_{t}=\pi \leq \xi<2 \pi$, Eq. (27) can be reduced to the elliptic form

$$
\begin{aligned}
& \pm \alpha_{1} \int_{\phi_{t}}^{\phi} \mathrm{d} \phi^{\prime}=\int_{\pi}^{\xi} \frac{\mathrm{d} \varphi}{\sqrt{1-k_{1}^{2} \sin ^{2} \varphi}} \\
& =\int_{0}^{\xi} \frac{\mathrm{d} \varphi}{\sqrt{1-k_{1}^{2} \sin ^{2} \varphi}}-\int_{0}^{\pi} \frac{\mathrm{d} \varphi}{\sqrt{1-k_{1}^{2} \sin ^{2} \varphi}},
\end{aligned}
$$

where

$\alpha_{1}=\sqrt{2 \sqrt{3} \eta u_{t}}, \quad k_{1}=\sqrt{\frac{2-\sqrt{3}}{4}}$.

So, we may write the solution for $\phi$ as

$\pm \alpha_{1} \phi=2 K\left(k_{1}\right)-F\left(\xi, k_{1}\right)$,

where $K(k)$ and $F(\psi, k)$ are the complete and incomplete elliptic integrals of the first kind, respectively, and we have assumed that $\phi_{t}=0$. Therefore, by using some formulas and identities of the Jacobian elliptic functions, we can write the equation of the trajectory as

$r(\phi, b)=\frac{r_{t}(b)}{1+\sqrt{3} \operatorname{tn}^{2}\left(\frac{\alpha_{1}(b) \phi}{2}, k_{1}\right) \operatorname{dn}^{2}\left(\frac{\alpha_{1}(b) \phi}{2}, k_{1}\right)}$,

where $\operatorname{tn}(x, k)$ and $\operatorname{dn}(x, k)$ are Jacobi's elliptic functions (see Appendix 1 and Refs. [45-47]). Obviously, this trajec- 


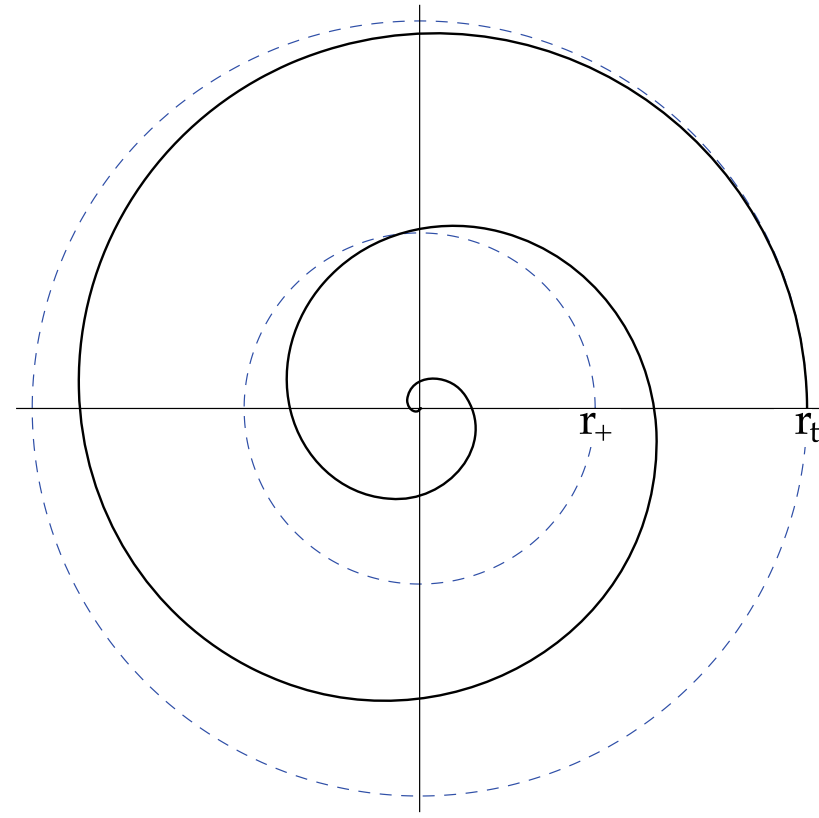

Fig. 4 Polar plot for a confined trajectory of photons from a distance $r_{t}$

tory depends on the impact parameter and is shown in Fig. 4 for photons falling from $r_{t}$.

\subsection{Unbounded motion}

We now consider the case when the impact parameter lies between $0<b<b_{c}$, which means that the real root is a negative one $r_{n} \equiv-r_{D}$, where $r_{D}$ is given by Eq. (26), so there is no turning point. Obviously, this negative root lacks physical interpretation, but it is important for determining the shape of the trajectory. Therefore, setting $u=1 / r$ again with $u_{D}=1 / r_{D}$, the equation of motion (19) can be written as

$$
\begin{aligned}
\frac{\mathrm{d} u}{\mathrm{~d} \phi} & = \pm \sqrt{2 \eta} \sqrt{u^{3}+u_{D}^{3}} \\
& = \pm \sqrt{2 \eta} \sqrt{\left(u+u_{D}\right)\left[\left(u-\frac{u_{D}}{2}\right)^{2}+\frac{3 u_{D}^{2}}{4}\right]} .
\end{aligned}
$$

In order to integrate Eq. (33), we now make the substitution

$u=u_{D}\left(\sqrt{3} \cot ^{2} \frac{\xi}{2}-1\right)$

where the range is

$$
\begin{aligned}
& u \rightarrow 0 \quad \text { (infinity), } \quad \xi_{\infty}=\arccos \left(-\frac{2-\sqrt{3}}{2}\right), \\
& u \rightarrow \infty \quad \text { (singularity), } \quad \xi_{s}=\pi,
\end{aligned}
$$

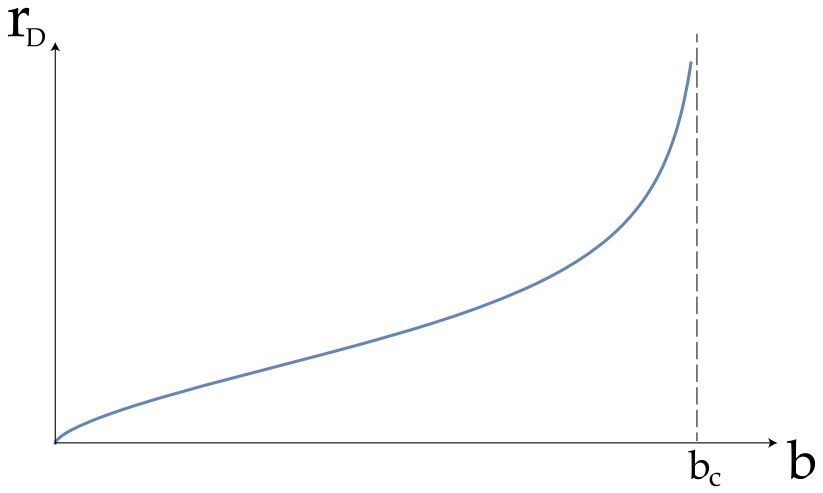

Fig. 5 Plot for the modulus of the real negative root $r_{D}$ as a function of the impact parameter associated with the unbounded motion of photons, i.e. $0<b<b_{c}$

so the quadrature becomes

$$
\begin{aligned}
\pm \alpha_{2} \int_{\phi_{\infty}}^{\phi} \mathrm{d} \phi^{\prime} & =\int_{\xi_{\infty}}^{\xi} \frac{\mathrm{d} \varphi}{\sqrt{1-k_{2}^{2} \sin ^{2} \varphi}} \\
& =\int_{0}^{\xi} \frac{\mathrm{d} \varphi}{\sqrt{1-k_{2}^{2} \sin ^{2} \varphi}}-\int_{0}^{\xi \infty} \frac{\mathrm{d} \varphi}{\sqrt{1-k_{2}^{2} \sin ^{2} \varphi}},
\end{aligned}
$$

with

$\alpha_{2}=\sqrt{2 \sqrt{3} \eta u_{D}}, \quad k_{2}=\sqrt{\frac{2+\sqrt{3}}{4}}$.

Note from Eqs. (30) and (38) that the module of one trajectory corresponds to the complementary module of the other, $k_{1}=\sqrt{1-k_{2}^{2}}=k_{2}^{\prime}$ and $k_{2}=\sqrt{1-k_{1}^{2}}=k_{1}^{\prime}$. Therefore, assuming that $\phi_{\infty}=0$ we may write

$\pm \alpha_{2} \phi=F\left(\xi, k_{2}\right)-F\left(\xi_{\infty}, k_{2}\right)$

which implies that the trajectory now is described by the polar equation

$r(\phi, b)=\frac{r_{D}(b)}{\sqrt{3} \operatorname{cs}^{2}\left(\Theta, k_{2}\right) \mathrm{nd}^{2}\left(\Theta, k_{2}\right)-1}$,

where $\operatorname{cs}(x, k)$ and $\operatorname{nd}(x, k)$ are Jacobi's elliptic functions (see Appendix 1), and the phase $\Theta$ is given by

$\Theta=\frac{F\left(\xi_{\infty}, k_{2}\right)-\alpha_{2}(b) \phi}{2}$

In Fig. 6 we have plotted the unbounded trajectory (40) for photons coming from infinity. 


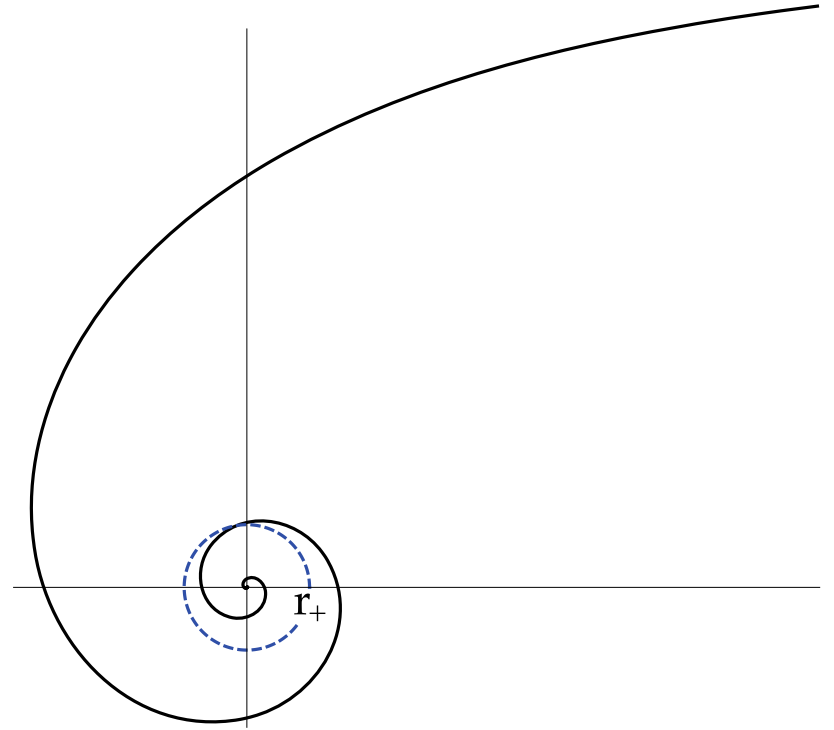

Fig. 6 Polar plot for the unbounded trajectory, Eq. (40), followed by photons whose impact parameter lies between $0<b<b_{c}$

\section{The Sagnac effect}

In this section we describe the Sagnac effect [48] by applying the formalism developed by Sakurai [49], Tartaglia [50], Rizzi and Ruggiero [51-54], among others, to the exterior space-time of the topological toroidal black hole. This approach was used to calculate this effect in the exterior space-time to an uncharged spherical symmetric black hole in conformal Weyl gravity [55]. For the non-rotating toroidal metric given by Eqs. (6) and (8) written in the usual Schwarzschild-like coordinates $\left(c t^{\prime}, r^{\prime}, \theta^{\prime}, \phi^{\prime}\right)$ :

$$
\begin{aligned}
\mathrm{d} s^{2}= & -\left(-\frac{2 \eta}{r^{\prime}}+\frac{r^{\prime 2}}{\ell^{2}}\right) c^{2} \mathrm{~d} t^{\prime 2}+\frac{\mathrm{d} r^{\prime 2}}{\left(-\frac{2 \eta}{r^{\prime}}+\frac{r^{\prime 2}}{\ell^{2}}\right)} \\
& +r^{\prime 2}\left(\mathrm{~d} \theta^{\prime 2}+\theta^{\prime 2} \mathrm{~d} \phi^{\prime 2}\right),
\end{aligned}
$$

so the transformation to the azimuthal frame of the rotating platform

$c t=c t^{\prime}, \quad r=r^{\prime}, \quad \theta=\theta^{\prime}, \quad \phi=\phi^{\prime}-\Omega t^{\prime}$,

where $\Omega$ is the constant angular velocity of the physical frame, yields the metric (after setting $r=R$ and $\theta=1$ )

$$
\begin{aligned}
\mathrm{d} s^{2}= & -\left(-\frac{2 \eta}{R}+\frac{R^{2}}{\ell^{2}}-\frac{\Omega^{2} R^{2}}{c^{2}}\right) \mathrm{d}(c t)^{2}+R^{2} \mathrm{~d} \phi^{2} \\
& +2 \frac{\Omega}{c} R^{2} \mathrm{~d} \phi \mathrm{d}(c t) .
\end{aligned}
$$

Therefore, the non-zero components of the unit vector $\gamma^{\alpha}$ along the trajectory $r=R$ are given by

$\gamma^{t}=\frac{1}{\sqrt{-g_{t t}}}=\gamma_{J}$

$$
\begin{aligned}
& \gamma_{t}=-\sqrt{-g_{t t}}=-\gamma_{J}^{-1}, \\
& \gamma_{\phi}=g_{\phi t} \gamma^{t}=\frac{\Omega}{c} R^{2} \gamma_{J},
\end{aligned}
$$

where

$\gamma_{J}=\left[R^{2}\left(\frac{1}{\ell^{2}}-\frac{\Omega^{2}}{c^{2}}\right)-\frac{2 \eta}{R}\right]^{-1 / 2}$.

In terms of this component, the gravito-electric and gravitomagnetic potentials are given by

$$
\begin{aligned}
& \phi^{G}=-c^{2} \gamma^{t}=-c^{2} \gamma_{J}, \\
& A_{\phi}^{G}=c^{2} \frac{\gamma_{\phi}}{\gamma_{t}}=-c \Omega R^{2} \gamma_{J}^{2} .
\end{aligned}
$$

As was shown in [51], it is possible to express the phase shift and time delay between light beams detected by a comoving observer on the interferometer in terms of the gravitomagnetic induction field, $\mathbf{B}^{G}=\nabla \times \mathbf{A}^{G}$, by means of the expressions

$\Delta \Phi=\frac{2 \epsilon \gamma_{t}}{\hbar c^{3}} \int_{S} \mathbf{B}^{G} \cdot \mathrm{d} \mathbf{S}=\frac{2 \epsilon \gamma_{t}}{\hbar c^{3}} \int_{\zeta(S)} \mathbf{A}^{G} \cdot \mathrm{d} \mathbf{r}$

and

$\Delta \tau=\frac{2 \gamma_{t}}{c^{3}} \int_{S} \mathbf{B}^{G} \cdot \mathrm{d} \mathbf{S}=\frac{2 \gamma_{t}}{c^{3}} \int_{\zeta(S)} \mathbf{A}^{G} \cdot \mathrm{d} \mathbf{r}$,

where $\epsilon$ is the relative energy of the photon as measured in the interferometer. Therefore, the phase shift turns out to be

$$
\Delta \Phi=\frac{4 \pi \epsilon}{\hbar c} \frac{\tilde{\Omega} R}{\sqrt{1-\tilde{\Omega}^{2}-\frac{r_{+}^{3}}{R^{3}}}},
$$

while the time delay turns out to be

$\Delta \tau=\frac{4 \pi}{c} \frac{\tilde{\Omega} R}{\sqrt{1-\tilde{\Omega}^{2}-\frac{r_{+}^{3}}{R^{3}}}}$,

where $\tilde{\Omega}=\Omega / \Omega_{\ell}$ is the dimensionless angular velocity, and $\Omega_{\ell} \equiv c / \ell$. Thus, from this last equation, we can see that if $\Omega=0$, i.e., performing a measure of the propagation time in a non-rotating frame, no Sagnac effect arises. Also, there is an upper limit for the angular velocity $\Omega_{R}$ related to the radius of the orbit $R$ (see Fig. 7) given by

$\Omega<\Omega_{R} \equiv \Omega_{\ell} \sqrt{1-\left(\frac{r_{+}}{R}\right)^{3}}$. 


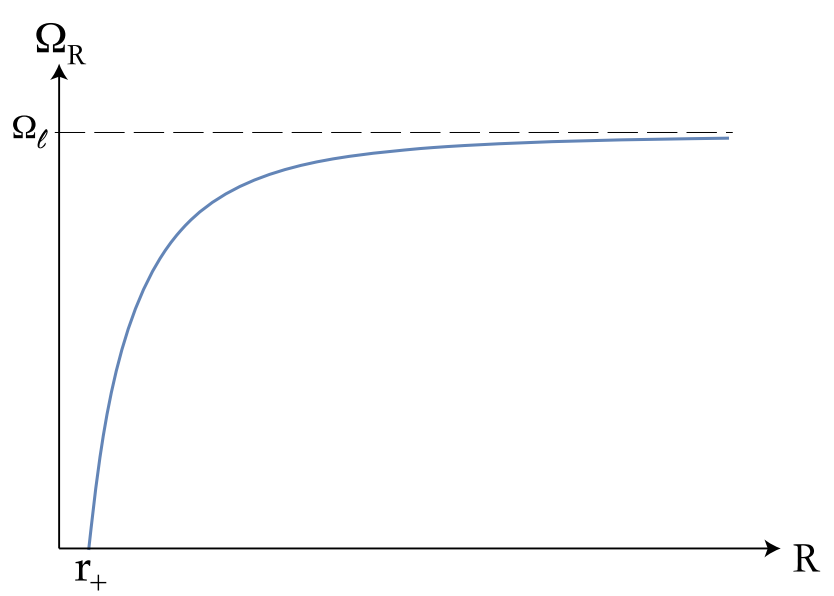

Fig. 7 Plot for the maximum angular velocity $\Omega_{R}$ as a function of the radius of the orbit $R$ for an interferometer in the Sagnac effect

\section{Summary}

In this paper we have studied the null structure of the geodesics for a toroidal topological space-time which surrounds a black hole in the conformal Weyl gravity. First, we obtained an explicit behavior of radial photons to conclude that, while no changes in the motion to the singularity with respect to the Schwarzschild anti-de Sitter counterpart is found, there is a non-trivial coordinate time $t_{\infty}$ for the description of the motion to the spatial infinity (see Eq. (24)). A similar result was obtained by Villanueva and Vásquez but in the context of the Lifshitz space-time [44]. Next, following the standard Lagrangian procedure, we have obtained analytically the trajectories of the confined and unbounded angular motion for photons in terms of Jacobi elliptic functions, Eqs. (32) and (40), and we have shown our results in Figs. 4 and 6 , respectively. Obviously, these trajectories depend on the impact parameter $b$ and, due to the topology, always fall to the singularity, which is a characteristic of AdS space-times. Finally, the Sagnac effect has been studied for this topological space-time. Our result is consistent with those obtained previously in other geometries in the sense that no Sagnac effect arises for a non-rotating frame. In addition, we have found a strong condition for its existence, which depends on the theory's parameters $\{\eta, \ell\}$ (in $r_{+}$and $\Omega_{\ell}$ ) as well as on the radius of the circular orbit $R$. This condition is given in Eq. (55); cf.

$\Omega<\Omega_{R} \equiv \Omega_{\ell} \sqrt{1-\left(\frac{r_{+}}{R}\right)^{3}}$,

for which the upper limit for the angular velocity $\Omega_{R}$ was plotted in Fig. 7 as a function of the radius $R$.

Finally, our study provides a simple physical visualization of the null trajectories and their main characteristics, and complements other studies carried out in the standard and/or trivial topology in conformal Weyl gravity [11-15], as well as in physically similar situations such as time-like geodesics on toroidal space-times [56] and motion of particles in toroidal magnetic fields on a Schwarzschild background [57], among others $[58,59]$.

Acknowledgements The authors acknowledge useful conversations with Prof. Dr. Ricardo Troncoso, Prof. Dr. Graeme Candlish and Dr. Helen Lowry. In addition, we acknowledge to Vicerrectoría de Investigación e Innovación of the Universidad de Valparaíso for support of this work.

Open Access This article is distributed under the terms of the Creative Commons Attribution 4.0 International License (http://creativecomm ons.org/licenses/by/4.0/), which permits unrestricted use, distribution, and reproduction in any medium, provided you give appropriate credit to the original author(s) and the source, provide a link to the Creative Commons license, and indicate if changes were made. Funded by SCOAP ${ }^{3}$.

\section{Appendix A: A brief review of Jacobian elliptic functions}

As a starting point, let us consider the elliptic integral [4547]

$$
\begin{aligned}
u(y, k) \equiv u & =\int_{0}^{y} \frac{d t}{\sqrt{\left(1-t^{2}\right)\left(1-k^{2} t^{2}\right)}} \\
& =\int_{0}^{\varphi} \frac{d \theta}{\sqrt{1-k^{2} \sin ^{2} \theta}}=F(\varphi, k),
\end{aligned}
$$

where $F(\varphi, k)$ is the normal elliptic integral of the first kind, and $k$ is the modulus. The problem of the inversion of this integral was studied and solved by Abel and Jacobi, and one defines the inverse function by $y=\sin \varphi=\operatorname{sn}(u, k)$ with $\varphi=\operatorname{am} u$, called the Jacobi elliptic sine $u$, and the amplitude $u$.

The function sn $u$ is an odd elliptic function of order two. It possesses a simple pole of residue $1 / k$ at every point congruent to $i K^{\prime}\left(\bmod 4 K, 2 i K^{\prime}\right)$ and a simple pole of residue $-1 / k$ at points congruent to $2 K+i K^{\prime}\left(\bmod 4 K, 2 i K^{\prime}\right)$, where $K \equiv K(k)=F(\pi / 2, k)$ is the complete elliptic integral of the first kind, $K^{\prime}=F\left(\pi / 2, k^{\prime}\right)$, and $k^{\prime}=\sqrt{1-k^{2}}$ is the complementary modulus.

Two other functions can then be defined by $\operatorname{cn}(u, k)=$ $\sqrt{1-y^{2}}=\cos \varphi$, which is called the Jacobi elliptic cosine $u$, and which is an even function of order two, and $\operatorname{dn}(u, k)=$ $\sqrt{1-k^{2} y^{2}}=\Delta \varphi=\sqrt{1-k^{2} \sin \varphi}$, called the Jacobi elliptic delta $u$, which is an even function. The set of functions $\{\operatorname{sn} u, \operatorname{cn} u, \operatorname{dn} u\}$ are called Jacobian elliptic functions, and they take the following special values:

$$
\begin{array}{ll}
\operatorname{sn}(u, 0)=\sin u, & \operatorname{sn}(u, 1)=\tanh u, \\
\operatorname{cn}(u, 0)=\cos u, & \operatorname{cn}(u, 1)=\operatorname{sech} u, \\
\operatorname{dn}(u, 0)=1, & \operatorname{dn}(u, 1)=\operatorname{sech} u, \\
\operatorname{tn}(u, 0)=\tan u, & \operatorname{tn}(u, 1)=\sinh u .
\end{array}
$$


The quotients and reciprocal of $\{\operatorname{sn} u, \operatorname{cn} u, \operatorname{dn} u\}$ are designated in Glaisher's notation by

$$
\begin{aligned}
& \operatorname{ns} u=\frac{1}{\operatorname{sn} u}, \quad \operatorname{cs} u=\frac{\operatorname{cn} u}{\operatorname{sn} u}, \quad \operatorname{ds} u=\frac{\operatorname{dn} u}{\operatorname{sn} u}, \\
& \operatorname{nc} u=\frac{1}{\operatorname{cn} u}, \quad \operatorname{tn} u \equiv \operatorname{sc} u=\frac{\operatorname{sn} u}{\operatorname{cn} u}, \quad \operatorname{dc} u=\frac{\operatorname{dn} u}{\operatorname{cn} u}, \\
& \operatorname{nd} u=\frac{1}{\operatorname{dn} u}, \quad \operatorname{sd} u=\frac{\operatorname{sn} u}{\operatorname{dn} u}, \quad \operatorname{cd} u=\frac{\operatorname{cn} u}{\operatorname{dn} u} .
\end{aligned}
$$

Therefore, in all, we have 12 Jacobian elliptic functions. Finally, some useful fundamental relations between Jacobian elliptic functions used throughout this work are

$$
\begin{aligned}
& \operatorname{sn}^{2} u+\operatorname{cn}^{2} u=1, \\
& \operatorname{dn}^{2} u+k^{2} \operatorname{sn}^{2} u=1, \\
& \operatorname{dn}^{2} u-k^{2} \operatorname{cn}^{2} u=k^{\prime 2}, \\
& \operatorname{cn}^{2} u+k^{\prime 2} \operatorname{sn}^{2} u=\operatorname{dn}^{2} u, \\
& \frac{1-\operatorname{cn} 2 u}{1+\operatorname{cn} 2 u}=\operatorname{tn}^{2} u \operatorname{dn}^{2} u, \\
& \operatorname{cn}(u \pm 2 K)=-\operatorname{cn} u .
\end{aligned}
$$

\section{References}

1. H. Weyl, Zur gravitationstheorie. Ann. Phys. 54, 117 (1917)

2. H. Weyl, Reine infinitesimalgeometrie. Math. Z. 2, 384 (1918)

3. H. Weyl, Gravitation und elektrizität, Sitz. Ber. Preuss. Ak. Wiss., 465 (1918)

4. R. Bach, Zur Weylschen relativitätstheorie und der Weylschen erweiterung des krümmenstensorsbegriffs. Math. Z. 9, 110 (1921)

5. R.J. Riegart, Birkhoff's theorem in conformal gravity. Phys. Rev. Lett. 53, 315 (1984)

6. P.D. Mennheim, D. Kazanas, Exact vacuum solution to conformal Weyl gravity and galactic rotation curves. Astrophys. J. 342, 635 (1989)

7. D. Kazanas, P.D. Mennheim, General structure of the gravitational equations of motion in conformal Weyl gravity. Astrophys. J. Suppl. 76, 431 (1991)

8. P.D. Mennheim, D. Kazanas, Solutions to the Reissner-Nordström, Kerr, and Kerr-Newman problems in fourth-order conformal Weyl gravity. Phys. Rev. D 44, 417 (1991)

9. P.D. Mennheim, D. Kazanas, Newtonian limit of conformal gravity and the lack of necessity of the second order Poisson equation. Gen. Rel. Grav. 26, 337 (1994)

10. A. Edery, M.B. Paranjape, Classical tests for Weyl gravity: deflection of light and time delay. Phys. Rev. D 58, 024011 (1998)

11. S. Pireaux, Light deflection in Weyl gravity: critical distances for photon paths. Class. Quant. Grav. 21, 1897 (2004)

12. S. Pireaux, Light deflection in Weyl gravity: constraints on the linear parameter. Class. Quant. Grav. 21, 4317 (2004)

13. J. Sultana, D. Kazanas, Bending of light in conformal Weyl gravity. Phys. Rev. D 81, 127502 (2010)

14. J. Sultana, D. Kazanas, J.L. Said, Conformal Weyl gravity and perihelion precession. Phys. Rev. D 86, 084008 (2012)

15. J.R. Villanueva, M. Olivares, On the null trajectories in conformal Weyl gravity. JCAP 06, 040 (2013)

16. J.L. Said, J. Sultana, K.Z. Adami, Gravitomagnetic effects in conformal gravity. Phys. Rev. D 88, 087504 (2013)

17. J.L. Said, J. Sultana, K.Z. Adami, Exact static cylindrical solution to conformal Weyl gravity. Phys. Rev. D 85, 104054 (2012)
18. H. Lu, Y. Pang, C.N. Pope, J.F. Vazquez-Poritz, AdS and Lifshitz black holes in conformal and Einstein-Weyl gravities. Phys. Rev. D 86, 044011 (2012)

19. F. Payandeh, M. Fathi, Spherical solutions due to the exterior geometry of a charged Weyl black hole. Int. J. Theor. Phys. 51, 2227 (2012)

20. S. Chandrasekhar, The Mathematical Theory of Black Holes (Oxford University Press, New York, 1983)

21. N. Cruz, M. Olivares, J.R. Villanueva, The geodesic structure of the Schwarzschild anti-de Sitter black hole. Class. Quant. Grav. 22, 1167-1190 (2005)

22. B. Shutz, A First Course in General Relativity (Cambridge University Press, Cambridge, 1990)

23. M. Olivares, J. Saavedra, C. Leiva, J.R. Villanueva, Motion of charged particles on the Reissner-Nordström (anti)-de Sitter black holes. Mod. Phys. Lett. A 26, 2923 (2011)

24. M.J. Jaklitsch, C. Hellaby, D.R. Matravers, Particle motion in the spherically symmetric vacuum solution with positive cosmological constant. Gen. Rel. Grav. 21, 941 (1989)

25. J.R. Villanueva, J. Saavedra, M. Olivares, N. Cruz, Photons motion in charged anti de Sitter black holes. Astrophys. Space Sci. 344, 437-446 (2013)

26. M. Halilsoy, O. Gurtug, S. Habib Mazharimousavi, Rindler modified Schwarzschild geodesics. Gen. Rel. Grav 45, 2363 (2013)

27. C. Leiva, J. Saavedra, J.R. Villanueva, The geodesic structure of the Schwarzschild black holes in gravity rainbow. Mod. Phys. Lett. A 24, 1443-1451 (2009)

28. D. Klemm, Topological black holes in Weyl conformal gravity. Class. Quant. Grav. 15, 3195 (1998)

29. R.G. Cai, Y.Z. Zhang, Black plane solutions in four-dimensional space-times. Phys. Rev. D 54, 4891 (1996)

30. R.G. Cai, J.Y. Ji, K.S. Soh, Topological dilaton black holes. Phys. Rev. D 57, 6547 (1998)

31. J. Kowalski-Glikman, D. Nowak-Szczepaniak, Topological black holes in quantum gravity. Phys. Lett. A 277, 83-86 (2000)

32. C. Martinez, J.P. Staforelli, R. Troncoso, Topological black holes dressed with a conformally coupled scalar field and electric charge. Phys. Rev. D 74, 044028 (2006)

33. O. Mišković, R. Olea, Topological regularization and self-duality in four-dimensional anti-de Sitter gravity. Phys. Rev. D 79, 124020 (2009)

34. L. Aránguiz, X. Kuang, O. Mišković, Topological black holes in pure Gauss-Bonnet gravity and phase transitions. Phys. Rev D 93, 064039 (2016)

35. F. Herrera, Y. Vásquez, AdS and Lifshitz black hole solutions in conformal gravity sourced with a scalar field. Phys. Lett. B 782, 305 (2018)

36. G. Panotopoulos, A. Rincón, Charged slowly rotating toroidal black holes in the $(1+3)$-dimensional Einstein-powerMaxwell theory. https://doi.org/10.1142/S0218271819500160. arXiv:1808.05171 [gr-qc]

37. J.P.S. Lemos, Two-dimensional black holes and planar general relativity. Class. Quant. Grav. 12, 1081 (1995)

38. C. Huang, C. Liang, A torus-like black hole. Phys. Lett. A 201, 27-32 (1995)

39. R.B. Mann, Pair production of topological anti-de Sitter black holes. Class. Quant. Grav. 14, L109 (1997)

40. D. Brill, J. Louko, P. Peldán, Thermodynamics of $(3+1)$ dimensional black holes with toroidal or higher genus horizons. Phys. Rev. D 56, 3600 (1997)

41. L. Vanzo, Black holes with unusual topology. Phys. Rev. D 56, 6475 (1997)

42. H. Maeda, G. Kunstatter, Exact time-dependent states for throat quantized toroidal AdS black holes. Phys. Rev. D 96, 106004 (2017) 
43. M. Astorino, F. Canfora, A. Giacomini, M. Ortaggio, Hairy AdS black hole with a toroidal horizon in 4D Einstein-nonlinear $\sigma$ model system. Phys. Lett. B 776, 236-241 (2018)

44. J.R. Villanueva, Y. Vásquez, About the coordinate time for photons in Lifshitz space-times. Eur. Phys. J. C 73(10), 2587 (2013)

45. P.F. Byrd, M.D. Friedman, Handbook of Elliptic Integrals for Engineers and Scientists, 2nd edn. (Springer, Berlin, 1971)

46. H. Hancock, Lectures on the Theory of Elliptic Functions (Dover Publications Inc., New York, 1958)

47. J.V. Armitage, W.F. Eberlein, Elliptic Functions. London Mathematical Society Student Texts, vol. 67 (Cambridge University Press, Cambridge, 2006)

48. M.G. Sagnac, C.R. Acad, Sci. Paris 141, 1220 (1905)

49. J.J. Sakurai, Comments on quantum mechanics interference due to the Earth's rotation. Phys. Rev. D 21, 2993-2994 (1980)

50. A. Tartaglia, General relativistic corrections to the Sagnac effect. Phys. Rev. D 58, 064009 (1998)

51. G. Rizzi, M.L. Ruggiero, The Sagnac phase shift suggested by the Aharonov-Bohm effect for relativistic matter beams. Gen. Rel. Grav. 35, 1745 (2003)

52. G. Rizzi, M.L. Ruggiero, A direct kinematical derivation of the relativistic Sagnac effect for light or matter beams. Gen. Rel. Grav. 35, 2129 (2003)
53. G. Rizzi, M.L. Ruggiero, Relativity in Rotating Frames, The Series Fundamental Theories of Physics (Kluwer Academic Publishers, Dordrecht, 2004)

54. M.L. Ruggiero, The Sagnac effect in curved space-times from an analogy with the Aharonov-Bohm effect. Gen. Rel. Grav. 37, 1845 (2005)

55. J. Sultana, The Sagnac effect in conformal Weyl gravity. Gen. Rel. Grav. 46, 1710 (2014)

56. S. B. P. Wickramasuriya, V. Joseph, K. I. S. Karunaratne, Geodesics in a toroidal space-time. arXiv:0411014

57. A.R. Prasanna, S. Sengupta, Charged particle trajectories in the presence of a toroidal magnetic field on a Schwarzschild background. Phys. Lett. A 193, 25 (1994)

58. G. Preti, On charged particle orbits in dipole magnetic fields around Schwarzschild black holes. Class. Quant. Grav. 21, 3433 (2004)

59. R. T. Jantzen, Geodesics on the torus and other surfaces of revolution clarified using undergraduate physics tricks with bonus: nonrelativistic and relativistic Kepler problems. arXiv:1212.6206 [math.DG] 